Human Embryology. The Development of Structure and Function.

2nd edition. By F Beck, D B Moffat, and D P Davies. (Figures + tables. £12.50.) Oxford: Blackwell Scientific Publications. 1985.

Although this volume is a second edition it has been given a new title and has been extensively rewritten. It is still, however, a text suitable for undergraduates beginning with a very basic outline of fundamental mechanisms and 'rules' of embryology. Then follow simplified but clear accounts of meiosis and mitosis, modes of inheritance, and physiology and histology of the male and female reproductive processes. The bulk of the book describes germ layer and early embryological development and then system and regional formation. Common malformations and their origins are covered 'en route'. The last three chapters are of a more general nature; the one discussing growth and development from conception to adolescence is excellent and informative, but the part of the penultimate chapter describing prenatal diagnosis is much too sketchy and should have been omitted, expanded, or a recent reference to a good review of the subject included.

The book is illustrated with clear line drawings throughout and a few clinical photographs. There is more text than in most basic embryology books, an advantage when trying to relate two dimensional diagrams to reality.

I have few criticisms to make. There is a tendency to use the words abnormalities, malformations, and deformities interchangeably when they should not be. Perhaps the need to keep the cost low prohibited the inclusion of light or electron microscopic illustrations of the embryology of, for example, the neural tube or palate, but it is a pity that up to date 'proof' of the line diagrams is not in this book.

I am sure those university teachers responsible for recommending books to medical undergraduates should seriously consider this as the basic embryology book. In addition it will be useful for those science or medical graduates wishing to remind themselves of basic embryology.

\section{Dian Donnal}

\section{Endocrine Genetics and Genetics of Growth}

Progress in Clinical and Biological Research Volume 200. Edited by C J Papadatos and C S Bartsocas. (Figures+tables. £49.00.) New York: Alan R. Liss. 1985.

The Fourth International Clinical Genetics Seminar was held in Athens in May 1985 and this book intended to provide an overall coverage of the subjects discussed. It also forms volume 200 of the series entitled Progress in Clinical and Biological Research. The editors and publishers are to congratulated for producing the volume so expeditiously.

An enormous range of subjects is covered by $\vec{z}$ contributions. The standard of contribution, unforte nately, also encompasses a wide range. Several topi⿸丆口广 are merely expanded case reports produced in pedantic fashion. One of the Greek joint editofs provides an interesting historical preamble to it lustrate the contribution of ancient Greek art an literature to topics such as goitres, dwarfs, and hermaphrodites. The genetics of normal growth $\underline{1} S$ presented in a clear and practical manner by that champion of human growth Professor Tanner. Op and co-workers complement this section by their account of growth analysis in clinical genetics, witho useful message on the differentiation between intrauterine growth retardation and congenital shortnes of stature. The only reference to DNA analysis in the book is to familial growth hormone deficien $\vec{E}^{y}$ caused by a $8.5 \mathrm{~kb}$ deletion which includes bo\% the growth hormone and human choriongc sommatomammatropin-like genes. The absence of any further contributions based on DNA analysiss and endocrine genetics is surprising.

Genetic thyroid diseases are adequately covered Koutras but the chapter is devoid of any new data ideas. Endemic cretinism fares worse, consisting of dull series of family pedigrees and unconvinciag clinical photographs. A succinct, up to date accountêf hereditary defects of adrenal steroidogenesis के provided by Prader, followed by Maria News summary of the HLA linkage studies in congenital adrenal hyperplasia. She now claims that the nonclassical variant of 21-hydroxylase deficiency (that premature adrenarche in children or polycys ovary-like syndrome in women) is the most frequeqr autosomal recessive genetic disorder in man. There are two chapters on sex chromosome abnormalities. A textbook account containing no original da赫 whatsoever is given of Turner's syndrome. In colltrast, Shirley Ratcliffe reports unique follow up dada on the Edinburgh Newborn Cytogenetic Survey, detailing longitudinal anthropometry on XXY, XY and $X X X$ groups. The final contribution is extensive and useful classification of pattern recog $\vec{P}$ ition in bone dysplasias.

This book contains quite a wealth of useful da However, there is also a surfeit of anecdotal case 